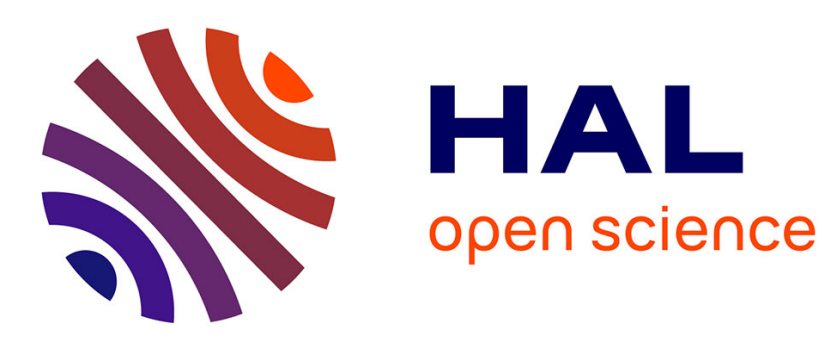

\title{
Global regularity for logarithmically critical 2D MHD equations with zero viscosity
}

\author{
Léo Agélas
}

\section{To cite this version:}

Léo Agélas. Global regularity for logarithmically critical 2D MHD equations with zero viscosity. Monatshefte für Mathematik, 2016, 181 (2), pp.245 - 266. 10.1007/s00605-016-0958-1 . hal-01380250

\author{
HAL Id: hal-01380250 \\ https://hal.science/hal-01380250
}

Submitted on 12 Oct 2016

HAL is a multi-disciplinary open access archive for the deposit and dissemination of scientific research documents, whether they are published or not. The documents may come from teaching and research institutions in France or abroad, or from public or private research centers.
L'archive ouverte pluridisciplinaire HAL, est destinée au dépôt et à la diffusion de documents scientifiques de niveau recherche, publiés ou non, émanant des établissements d'enseignement et de recherche français ou étrangers, des laboratoires publics ou privés. 


\title{
Global regularity for logarithmically critical 2D MHD equations with zero viscosity
}

\author{
Léo Agélas *
}

February 17, 2016

\begin{abstract}
In this article, the two-dimensional magneto-hydrodynamic (MHD) equations are considered with only magnetic diffusion. Here the magnetic diffusion is given by $\mathfrak{D}$ a Fourier multiplier whose symbol $m$ is given by $m(\xi)=|\xi|^{2} \log \left(e+|\xi|^{2}\right)^{\beta}$. We prove that there exists an unique global solution in $H^{s}\left(\mathbb{R}^{2}\right)$ with $s>2$ for these equations when $\beta>1$. This result improves the previous works which require that $m(\xi)=|\xi|^{2 \beta}$ with $\beta>1$ and brings us closer to the resolution of the well-known global regularity problem on the 2D MHD equations with standard Laplacian magnetic diffusion, namely $m(\xi)=|\xi|^{2}$.
\end{abstract}

Keywords MHD; Navier-Stokes, Euler, BKM's criterion.

Mathematics Subject Classification 76W05, 35Q35, 35Q60, 76B03.

\section{Introduction}

Magneto-hydrodynamics equations (MHD) describes the evolution of an electrically conducting fluid. Examples of such fluids include plasmas, liquid metals, and salt water or electrolytes. The field of MHD was initiated by Hannes Alfvén [1], for which he received the Nobel Prize in Physics in 1970. The fundamental concept behind MHD is that magnetic fields can induce currents in a moving conductive fluid, which in turn creates forces on the fluid and also changes the magnetic field itself. Due to their prominent roles in modeling many phenomena in astrophysics, geophysics and plasma physics, the MHD equations have been studied extensively mathematically.

More recent work on the MHD equations develops regularity criteria in terms of the velocity field and deals with the MHD equations with dissipation and magnetic diffusion given by general Fourier multiplier operators such as the fractional Laplacian operators (see [21, 22, 23, 8, 20, 13, 6, 24, 25]), well known also under the name of Generalized MHD (GMHD) equations. These equations are given by,

$$
\begin{cases}\partial_{t} u+(u \cdot \nabla) u+\nabla p+\nu(-\Delta)^{\alpha} u & =(b \cdot \nabla) b \\ \partial_{t} b+(u \cdot \nabla) b-(b \cdot \nabla) u+\eta(-\Delta)^{\beta} b & =0 \\ \nabla \cdot u=0, \nabla \cdot b=0 & \\ u(x, 0)=u_{0}, b(x, 0)=b_{0} & \end{cases}
$$

where $\alpha \geq 0, \beta \geq 0, \nu \geq 0$ and $\eta \geq 0$.

Among all the regularity criteria, one of particular interest is the Beale-Kato-Majda's criterion well known for Euler equations, extended in [5] to the ideal MHD equations, under the assumption on both velocity

\footnotetext{
*Department of Mathematics, IFP Energies nouvelles, 1 \& 4 avenue Bois Préau, 92852 Rueil-Malmaison Cedex, France (leo.agelas@ifpen.fr)
} 
field and magnetic field: $\int_{0}^{T}\left(\|\omega(t)\|_{L^{\infty}}+\|j(t)\|_{L^{\infty}}\right) d t<\infty$, where the vorticity $\omega=\nabla \times u$ and the density $j=\nabla \times b$. And so, the Beale-Kato-Majda's criterion ensures that the solution $(u, b)$ of the ideal MHD equations is smooth up to time $T$.

Meanwhile the two-dimensional (2D) Euler equation is globally well-posed for smooth initial data, however for the 2D inviscid MHD equations ( $\nu=0$ and $\eta=0$ in (1)), the global wellposedness of classical solution is still a big open problem. So the 2D GMHD equations has attracted much interest of many mathematicians and has motivated a large number of research papers concerning various generalizations and improvements.

For instance for $\nu=0$, to obtain the global regularity result:

Tran, Yu and Zhai [20] showed that $\beta>2$ suffices. Later Jiu and Zhao [13] and Yamazaki [24] independently improved the previous result with $\beta>\frac{3}{2}$. Then, Jiu and Zhao [14] and Cao, Wu and Yuan [6] independently showed in fact that $\beta>1$ suffices.

On the other hand, for $\beta=1$ and $\nu>0$, to obtain the global regularity result:

Tran, Yu and Zhai [20] showed that $\alpha \geq \frac{1}{2}$ suffices. Then, Yamazaki [25] obtained a better result with $\alpha>\frac{1}{3}$. Later, Ye and Xu [26] showed that $\alpha \geq \frac{1}{4}$ suffices. Then, Fan and al. [10] improved the previous result with the condition $0<\alpha \leq \frac{1}{2}$.

Thus, despite these recent developments, the global regularity issue of 2D GMHD system in the case $\nu=0$ and $\beta=1$ remains a challenging open problem up to date. The main reason for the unavailability of a proof of global regularity for the system of equations (1) in the case where $\nu=0$ and $\beta=1$ is due to the quadratic coupling between $u$ and $b$ which invalidates the vorticity conservation. Indeed, the structure of the vorticity is instantaneously altered due to the effects of the magnetic fields. This fact is the source of the main difficulty connected to the global existence of classical solutions, where no strong global a priori estimates are known till now. This difficulty is revealed through the equations of the ideal 2D MHD governing the vorticity $\omega=\partial_{1} u_{2}-\partial_{2} u_{1}$ and the current density $j=\partial_{1} b_{2}-\partial_{2} b_{1}$,

$$
\left\{\begin{array}{l}
\partial_{t} \omega+u \cdot \nabla \omega=b \cdot \nabla j \\
\partial_{t} j+u \cdot \nabla j=b \cdot \nabla \omega+T(\nabla u, \nabla b)
\end{array}\right.
$$

where,

$$
T(\nabla u, \nabla b)=2 \partial_{1} b_{1}\left(\partial_{2} u_{1}+\partial_{1} u_{2}\right)+2 \partial_{2} u_{2}\left(\partial_{2} b_{1}+\partial_{1} b_{2}\right) .
$$

We observe that the magnetic field contributes in the last nonlinear part of the second equation with the quadratic term $T(\nabla u, \nabla b)$.

Then, in this paper, we consider the initial-value problem for the 2D incompressible magneto-hydrodynamics equations with Fourier multiplier operators magnetic diffusion,

$$
\begin{cases}\partial_{t} u+(u \cdot \nabla) u & =-\nabla p+(b \cdot \nabla) b \\ \partial_{t} b+(u \cdot \nabla) b+\mathfrak{D} b & =(b \cdot \nabla) u \\ \nabla \cdot u=0, \nabla \cdot b=0 & \\ u(x, 0)=u_{0}, b(x, 0)=b_{0}, & \end{cases}
$$

where $\mathfrak{D}$ is a Fourier multiplier whose symbol $m: \mathbb{R}^{2} \mapsto \mathbb{R}^{+}$is non-negative; the case $m(\xi)=|\xi|^{2}$ reduces to the MHD equations with Laplacian magnetic diffusion corresponding to $\nu=0$ and $\beta=1$ in (1), which models many significant phenomena such as the magnetic reconnection in astrophysics and geomagnetic dynamo in geophysics (see [18]), while the case $m=0$ is the ideal MHD system. The problem of global well-posedness of the two-dimensional MHD equations with partial dissipation and magnetic diffusion has generated considerable interest recently [7, 3, 12, 17, 27], and remains highly challenging. Thus, the problem of uniqueness and global regularity of 2D MHD system (3) for the case $m(\xi)=|\xi|^{2}$ remains widely open, but recently there has been some progress made. Indeed, this problem have been solved independently in $[6,14]$ for the case $m(\xi)=|\xi|^{2 \beta}$ with $\beta>1$.

In this paper, we improve their result by obtaining uniqueness and global regularity for the case,

$$
m(\xi) \equiv|\xi|^{2} \log \left(e+|\xi|^{2}\right)^{\beta}, \text { with } \beta>1 .
$$


Thus

$$
\mathfrak{D}=(-\Delta) \log (e-\Delta)^{\beta} .
$$

More precisely, we prove the following theorem,

Theorem 1.1 Assume that $\left(u_{0}, b_{0}\right) \in H^{s}\left(\mathbb{R}^{2}\right)$ with $s>2, \nabla \cdot u_{0}=0, \nabla \cdot b_{0}=0$. Then (3) with $\mathfrak{D}$ given by (5) has a unique global solution $(u, b)$ satisfying, for any $T>0$,

$$
(u, b) \in C\left([0, T] ; H^{s}\left(\mathbb{R}^{2}\right)\right), \quad \nabla j \in L^{1}\left([0, T] ; L^{\infty}\left(\mathbb{R}^{2}\right)\right),
$$

where $j=\nabla \times b$.

To this end, we take advantage of the approach used in [10] based on the properties of heat equation by using singular integral representations of equations (3). Our approach is similar to the one used in [14] but different from the one involved in [6] which is based on energy estimates and for which it seemed not possible to extend it to our borderline case. Further, our proof differs from the one given in [14].

Indeed, the proof given in [14] exploits the fact that initially for the bordeline case $\beta=1$ where then $\mathfrak{D}=-\Delta$, one can get uniform bound for $\left\|\nabla^{2} b\right\|_{L^{q}\left([0, T] ; L^{p}\left(\mathbb{R}^{2}\right)\right)}$ and $\|\omega\|_{L^{\infty}\left([0, T] ; L^{p}\left(\mathbb{R}^{2}\right)\right)}$ for some $p, q \in$ $[2, \infty$ [ by using some estimates for linear Stokes system (see [11]) and transport equations. Then by considering the operator $\mathfrak{D}=(-\Delta)^{\beta}$ with $\beta>1$, the authors were able to get better than the estimate on $\left\|\nabla^{2} b\right\|_{L^{2}\left([0, T] ; L^{p}\left(\mathbb{R}^{2}\right)\right)}$ by obtaining an estimate on $\left\|\nabla^{2+\delta} b\right\|_{L^{2}\left([0, T] ; L^{p}\left(\mathbb{R}^{2}\right)\right)}$ for some $\delta>0$. Hence, by using Sobolev embeddings, the authors obtained an uniform bound on $\|\nabla j\|_{L^{2}\left([0, T] ; L^{\infty}\left(\mathbb{R}^{2}\right)\right)}$ and then they got an uniform bound for $\|\omega\|_{L^{2}\left([0, T] ; L^{\infty}\left(\mathbb{R}^{2}\right)\right)}$ deriving from estimates for transport equations (see for instance Lemma 4.1 in [15]).

However in our case where $\mathfrak{D}=(-\Delta) \log (e-\Delta)^{\beta}$ with $\beta>1$, it was no longer possible to proceed as previously, we then overcome this difficulty by establishing and using a series of estimates on the semigroup generated by $-\mathfrak{D}$, that is $e^{-t \mathfrak{D}}$. This latter is used to get the solutions of equation (6).

Further, to get the proof of Theorem 1.1, it sufficed to show that for any $T>0, \int_{0}^{T}\|(\omega, j)(\tau)\|_{L^{\infty}} d \tau$ remains bounded. Indeed, this result follows from a Beale-Kato-Madja's (BKM) criterion (well-known for Euler and Navier-Stokes equations, see [2]) which states that: any solution $(u, b) \in C\left(\left[0, T\left[, H^{s}\left(\mathbb{R}^{2}\right)\right)\right.\right.$ of the MHD system (5) continues to belong up to the time $T$ to $C\left([0, T], H^{s}\left(\mathbb{R}^{2}\right)\right)$ under the assumption that $\int_{0}^{T}\|(\omega, j)(\tau)\|_{L^{\infty}} d \tau<\infty$, where $\omega, j$ are respectively the vorticity of $u, b$. One can also notice that this BKM's criterion applies also to the ideal MHD system.

Further, in Section 5, we have extended the BKM's criterion obtained in [5] for any integer $s \geq 3$ to all $s>2$. This improvement is obtained by using the logarithmic Sobolev inequality proved in $[16,15]$ which requires only that $s>2$ instead to use the one proved in [2] as it is the case in [5] and which requires that $s \geq 3$.

Then, we have proceeded in three steps for the proof of Theorem 1.1.

- Step 1 : Using a $H^{1}$-bound on $(u, b)$ proved in $[20,17]$ and using the singular integral representation of $b$ obtained from the second equation of (3), we get $b \in L^{\infty}\left(\mathbb{R}^{2} \times[0, T]\right)$ in Lemma 5.1.

- Step 2 : Then, after introducing the vorticity of $b, j=\nabla \times b$, we write the singular integral representation of $j$ to obtain a bound for $\int_{0}^{T}\|j(\tau)\|_{L^{\infty}} d \tau$ in Lemma 5.2, thanks to the previous result.

- Step 3 : After, writing the equation satisfied by the vorticity of $u, \omega=\nabla \times u$, we deduce that $\|\omega(t)\|_{L^{\infty}} \leq\left\|\omega_{0}\right\|_{L^{\infty}}+\|b\|_{L^{\infty}\left(\mathbb{R}^{2} \times[0, T]\right)} \int_{0}^{t}\|\nabla j\|_{L^{\infty}}$. Then after writing the singular integral representation of $\nabla j$ and using the previous results, we obtain a bound for $\int_{0}^{T}\|\nabla j\|_{L^{\infty}}$ in Lemma 5.3. As a consequence, we infer a bound on $\|\omega\|_{L^{\infty}\left(\mathbb{R}^{2} \times[0, T]\right)}$. Then, gathering all the results, we obtain that for any $T>0, \int_{0}^{T}\|(\omega, j)(\tau)\|_{L^{\infty}} d \tau$ remains bounded.

To obtain these results, we have used a series of Lemmata given in Section 3, which gives estimates in some Sobolev spaces of the kernel $K$ involved in the singular integral representation. 
The paper is organized as follows. In section 2, we give some notations and introduce the functional spaces. In section 3, we give some estimates on the kernel $K$. In section 4 , we establish the local wellposedness of the Cauchy problem of the partially viscous magneto-hydrodynamic system (3) and give a characterization of the maximal time existence of strong solutions. In section 5, we prove Theorem 1.1 by showing that for any $T>0, \int_{0}^{T}\|(\omega, j)(\tau)\|_{L^{\infty}} d \tau$ remains bounded.

\section{Some notations}

We denote $A \lesssim B$, the estimate $A \leq C B$ where $C>0$ is a constant.

We denote by $B C$ the class of bounded and continuous functions and by $B C^{m}$ the class of bounded and $m$ times continuously derivable functions.

For any $f \in L^{p}\left(\mathbb{R}^{2}\right)$, with $1 \leq p \leq \infty$, we denote by $\|f\|_{p}$ and $\|f\|_{L^{p}}$, the $L^{p}$-norm of $f$.

Given an absolutely integrable function $f \in L^{1}\left(\mathbb{R}^{2}\right)$, we define the Fourier transform $\hat{f}: \mathbb{R}^{2} \longmapsto \mathbb{C}$ by the formula,

$$
\hat{f}(\xi)=\int_{\mathbb{R}^{2}} e^{-2 \pi i x \cdot \xi} f(x) d x
$$

and extend it to tempered distributions. We will use also the notation $\mathcal{F}(f)$ for the Fourier transform of $f$. We define also the inverse Fourier transform $\check{f}: \mathbb{R}^{2} \longmapsto \mathbb{C}$ by the formula,

$$
\check{f}(x)=\int_{\mathbb{R}^{2}} e^{2 \pi i x \cdot \xi} f(\xi) d \xi .
$$

For $s \in \mathbb{R}$, we define the Sobolev norm $\|f\|_{H^{s}\left(\mathbb{R}^{2}\right)}$ of a tempered distribution $f: \mathbb{R}^{2} \longmapsto \mathbb{R}$ by,

$$
\|f\|_{H^{s}\left(\mathbb{R}^{2}\right)}=\left(\int_{\mathbb{R}^{2}}\left(1+|\xi|^{2}\right)^{s}|\hat{f}(\xi)|^{2} d \xi\right)^{\frac{1}{2}}
$$

and then we denote by $H^{s}\left(\mathbb{R}^{2}\right)$ the space of tempered distributions with finite $H^{s}\left(\mathbb{R}^{2}\right)$ norm, which matches when $s$ is a non negative integer with the classical Sobolev space $H^{k}\left(\mathbb{R}^{2}\right), k \in \mathbb{N}$. The Sobolev space $H^{s}\left(\mathbb{R}^{2}\right)$ can be written as $H^{s}\left(\mathbb{R}^{2}\right)=J^{-s} L^{2}\left(\mathbb{R}^{2}\right)$ where $J=(1-\Delta)^{\frac{1}{2}}$.

For $s>-1$, we also define the homogeneous Sobolev norm,

$$
\|f\|_{\dot{H}^{s}\left(\mathbb{R}^{2}\right)}=\left(\int_{\mathbb{R}^{2}}|\xi|^{2 s}|\hat{f}(\xi)|^{2} d \xi\right)^{\frac{1}{2}},
$$

and then we denote by $\dot{H}^{s}\left(\mathbb{R}^{2}\right)$ the space of tempered distributions with finite $\dot{H}^{s}\left(\mathbb{R}^{2}\right)$ norm. We use the Fourier transform to define the fractional Laplacian operator $(-\Delta)^{\alpha},-1<\alpha \leq 1$. We define it as follows,

$$
\widehat{(-\Delta)^{\alpha}} f(\xi)=|\xi|^{2 \alpha} \hat{f}(\xi) .
$$

We denote by $\mathbb{P}$ the projector onto divergence free vector fields given by $\mathbb{P}=\operatorname{Id}-\nabla \Delta^{-1}$ div.

\section{Some estimates}

In this section, for a given $t>0$, we consider the inverse Fourier transform of $e^{-t m(\xi)}$, where $m$ is given by (4), namely $K(x, t)=\mathcal{F}^{-1}\left(e^{-t m(\xi)}\right)(x)$. The kernel $K$ allows to write the singular integral representation of the solution of equations of type,

$$
\begin{cases}\partial_{t} v+\mathfrak{D} v & =f \\ v(0) & =v_{0}\end{cases}
$$


namely, we have $v(t)=K(t) \star v_{0}+\int_{0}^{t} K(t-s) \star f(s) d s$. For the proofs given in Section 5 , it was crucial to get estimates of $K(t)$ in $\dot{H}^{s}$ and also in $W^{m, 1}$ for $m \in\{0,1,2\}$. All theses estimates derive from the following Proposition,

Proposition 3.1 For any $n \in \mathbb{N}, s>n-1$, there exists a real $C>0$ depending only on $s, n$ such that for all $t>0$,

$$
J_{s, n}(t):=\int_{\mathbb{R}^{2}}|\xi|^{2 s} \log \left(e+|\xi|^{2}\right)^{n \beta} e^{-2 t|\xi|^{2} \log \left(e+|\xi|^{2}\right)^{\beta}} \leq \frac{C}{t^{s+1} \log \left(e+\frac{1}{t \log \left(e+\frac{1}{t}\right)^{\beta}}\right)^{\beta(s-n+1)}} .
$$

Proof. We have for any $R>0$,

$J_{s, n}(t)=\int_{|\xi|^{2} \leq R}|\xi|^{2 s} \log \left(e+|\xi|^{2}\right)^{n \beta} e^{-2 t|\xi|^{2} \log \left(e+|\xi|^{2}\right)^{\beta}} d \xi+\int_{|\xi|^{2}>R}|\xi|^{2 s} \log \left(e+|\xi|^{2}\right)^{n \beta} e^{-2 t|\xi|^{2} \log \left(e+|\xi|^{2}\right)^{\beta}} d \xi$.

For the first term at the right hand side of equation (7), we have,

$$
\begin{aligned}
|\xi|^{2 s} \log \left(e+|\xi|^{2}\right)^{n \beta} e^{-2 t|\xi|^{2} \log \left(e+|\xi|^{2}\right)^{\beta}} & =|\xi|^{2(s-n)}\left(|\xi|^{2} \log \left(e+|\xi|^{2}\right)^{\beta}\right)^{n} e^{-2 t|\xi|^{2} \log \left(e+|\xi|^{2}\right)^{\beta}} \\
& \leq|\xi|^{2(s-n)} \sup _{r \geq 0} r^{n} e^{-2 t r} \\
& \leq C_{n} \frac{|\xi|^{2(s-n)}}{t^{n}}
\end{aligned}
$$

where $C_{n}=1$ if $n=0$ else $C_{n}=\frac{n^{n} e^{-n}}{2^{n}}$. Then, we deduce,

$$
\begin{aligned}
\int_{|\xi|^{2} \leq R}|\xi|^{2 s} \log \left(e+|\xi|^{2}\right)^{n \beta} e^{-2 t|\xi|^{2} \log \left(e+|\xi|^{2}\right)^{\beta}} d \xi & \leq \frac{C_{n}}{t^{n}} \int_{|\xi| \leq \sqrt{R}}|\xi|^{2(s-n)} d \xi \\
& =\frac{\pi C_{n}}{s-n+1} \frac{R^{s-n+1}}{t^{n}} .
\end{aligned}
$$

For the second term at the right hand side of equation (7), we set $A=t \log (e+R)^{\beta}$ and we observe for all $|\xi|^{2}>R$

$$
\begin{aligned}
|\xi|^{2 s} \log \left(e+|\xi|^{2}\right)^{n \beta} e^{-2 t|\xi|^{2} \log \left(e+|\xi|^{2}\right)^{\beta}} & \leq|\xi|^{2 s} \log \left(e+|\xi|^{2}\right)^{n \beta} e^{-t|\xi|^{2} \log \left(e+|\xi|^{2}\right)^{\beta}} e^{-|\xi|^{2} A} \\
& \leq 2^{n} C_{n} \frac{|\xi|^{2(s-n)}}{t^{n}} e^{-|\xi|^{2} A}
\end{aligned}
$$

where for the last inequality, we have used (8) by replacing $t$ by $\frac{t}{2}$. Then, we obtain,

$$
\begin{aligned}
\int_{|\xi|^{2}>R}|\xi|^{2 s} \log \left(e+|\xi|^{2}\right)^{n \beta} e^{-2 t|\xi|^{2} \log \left(e+|\xi|^{2}\right)^{\beta}} d \xi & \leq \frac{2^{n} C_{n}}{t^{n}} \int_{\mathbb{R}^{2}}|\xi|^{2(s-n)} e^{-A|\xi|^{2}} d \xi \\
& =\frac{2^{n} C_{n}}{t^{n}} \frac{\widetilde{C}_{s, n}}{A^{s-n+1}} \widetilde{C}_{s, n} \\
& =\frac{2^{n} C_{n}}{t^{s+1}} \frac{\log (e+R)^{\beta(s-n+1)}}{\log }
\end{aligned}
$$

where $\widetilde{C}_{s, n}=\int_{\mathbb{R}^{2}}|y|^{2(s-n)} e^{-|y|^{2}} d y$.

Using (9) and (11), we deduce that there exists a real $C_{s, n}>0$ depending only on $s, n$ such that,

$$
J_{s, n}(t) \leq C_{s, n}\left(\frac{R^{s-n+1}}{t^{n}}+\frac{1}{t^{s+1} \log (e+R)^{\beta(s-n+1)}}\right) .
$$


We set,

$$
R=\frac{1}{t \log \left(e+\frac{1}{t}\right)^{\beta}}
$$

Then, from (12), we deduce,

$$
\begin{aligned}
J_{s, n}(t) & \leq \frac{C_{s, n}}{t^{s+1}}\left(\frac{1}{\log \left(e+\frac{1}{t}\right)^{\beta(s-n+1)}}+\frac{1}{\log \left(e+\frac{1}{t \log \left(e+\frac{1}{t}\right)^{\beta}}\right)^{\beta(s-n+1)}}\right) \\
& \leq \frac{2 C_{s, n}}{t^{s+1} \log \left(e+\frac{1}{t \log \left(e+\frac{1}{t}\right)^{\beta}}\right)^{\beta(s-n+1)}},
\end{aligned}
$$

which concludes the proof.

As a consequence of Proposition 3.1, we obtain the following Lemma,

Lemma 3.1 For all $s \geq 0$, there exists a real $C>0$ depending only on $s$ such that for all $t>0$,

$$
\|K(t)\|_{\dot{H}^{s}} \leq \frac{C}{t^{\frac{s+1}{2}} \log \left(e+\frac{1}{t \log \left(e+\frac{1}{t}\right)^{\beta}}\right)^{\frac{\beta(s+1)}{2}} .}
$$

Proof. We observe,

$$
\begin{aligned}
\|K(t)\|_{\dot{H}^{s}}^{2} & =\int_{\mathbb{R}^{2}}|\xi|^{2 s}|\widehat{K}(\xi, t)|^{2} d \xi \\
& =\int_{\mathbb{R}^{2}}|\xi|^{2 s} e^{-2 t|\xi|^{2} \log \left(e+|\xi|^{2}\right)^{\beta}} d \xi \\
& =J_{s, 0}(t),
\end{aligned}
$$

and thanks to Proposition 3.1, we conclude the proof.

From Lemma 3.1, we get the following Lemma.

Lemma 3.2 There exists a constant $C>0$ such that for all $t>0$,

$$
\|K(t)\|_{L^{\infty}} \leq \frac{C}{t \log \left(e+\frac{1}{t \log \left(e+\frac{1}{t}\right)^{\beta}}\right)^{\beta}} .
$$

Proof. Thanks to Gagliardo-Nirenberg inequality, we have,

$$
\|K(t)\|_{L^{\infty}} \lesssim\|K(t)\|_{L^{2}}^{\frac{1}{2}}\|K(t)\|_{\dot{H}^{2}}^{\frac{1}{2}},
$$

and thanks to Lemma 3.1 used with $s=0$ and $s=2$, we conclude the proof.

Now, we give the main Lemma of this Section. The main difficulty to establish Lemma 3.3, comes from the fact we do not have in $\mathbb{R}^{2}$ a Gagliardo-Nirenberg inequality of type $\left\|\nabla^{2} f\right\|_{L^{1}} \lesssim\left\|\nabla^{j} f\right\|_{L^{2}}^{a}\|f\|_{L^{2}}^{1-a}$, $j>2$.

Lemma 3.3 Let $m \in\{0,1,2\}$. There exists a real $C>0$ depending only on $\beta$ such that for all $t>0$,

$$
\left\|\nabla^{m} K(t)\right\|_{L^{1}} \leq \frac{C}{t^{\frac{m}{2}} \log \left(e+\frac{1}{t \log \left(e+\frac{1}{t}\right)^{\beta}}\right)^{\frac{\beta m}{2}}} .
$$


Proof. Since for any radial function $f$ in $\mathbb{R}^{2}$, we have $\check{f}(x)=\hat{f}(x)$, then we get $f(x)=\hat{h}(x)$, where $h=\hat{f}$. Since $\widehat{K}(\cdot, t)$ is a radial function, then we infer that $K(\cdot, t)$ is also a radial function, therefore we have, $K(x, t)=\widehat{G}(x, t)$, where $G(\xi, t)=\widehat{K}(\xi, t)$ and then,

$$
\left\|\nabla^{m} K(t)\right\|_{L^{1}}=\left\|\nabla^{m} \widehat{G}(t)\right\|_{L^{1}},
$$

For all $f \in H^{2}\left(\mathbb{R}^{2}\right)$, we get the following interpolation inequality,

$$
\|\hat{f}\|_{L^{1}} \lesssim\|f\|_{L^{2}}^{\frac{1}{2}}\|f\|_{\dot{H}^{2}}^{\frac{1}{2}}
$$

We denote by $\xi^{2}$ the matrix $\xi \otimes \xi$. Then, for any $t>0$, we get,

$$
\begin{aligned}
\left\|\nabla^{m} \widehat{G}(t)\right\|_{L^{1}} & =\left\|\mathcal{F}\left(\xi^{m} G(\xi, t)\right)\right\|_{L^{1}} \\
& \leq\left\|\xi^{m} G(\xi, t)\right\|_{L^{2}}^{\frac{1}{2}}\left\|\xi^{m} G(\xi, t)\right\|_{\dot{H}^{2}}^{\frac{1}{2}} \\
& =\left\||\xi|^{m} \widehat{K}(\xi, t)\right\|_{L^{2}}^{\frac{1}{2}}\left\|\nabla_{\xi}^{2}\left(\xi^{m} \widehat{K}(\xi, t)\right)\right\|_{L^{2}}^{\frac{1}{2}} \\
& =\|K\|_{\dot{H}^{m}}^{\frac{1}{2}}\left\|\nabla_{\xi}^{2}\left(\xi^{m} \widehat{K}(\xi, t)\right)\right\|_{L^{2}}^{\frac{1}{2}} .
\end{aligned}
$$

Since $m \in\{0,1,2\}$, we observe that there exists a constant $C>0$ such that for all $\xi \in \mathbb{R}^{2}, \xi \neq 0$,

$$
\left.\left|\nabla_{\xi}^{2}\left(\xi^{m} \widehat{K}(\xi, t)\right)\right| \leq\left. C\left(\delta_{m, 2} \mid \widehat{K}(\xi, t)\right)|+| \xi\right|^{m-1}\left|\nabla_{\xi} \widehat{K}(\xi, t)\right|+|\xi|^{m}\left|\nabla_{\xi}^{2} \widehat{K}(\xi, t)\right|\right),
$$

where $\delta_{m, 2}=0$ if $m \neq 2$ and $\delta_{m, 2}=1$ if $m=2$.

Since

$$
\widehat{K}(\xi, t)=e^{-t|\xi|^{2} \log \left(e+|\xi|^{2}\right)^{\beta}},
$$

then after elementary computations, we deduce that there exists a real $C_{\beta}>0$ depending only on $\beta$ such that for all $\xi \in \mathbb{R}^{2}$,

$$
\begin{aligned}
\left|\nabla_{\xi} \widehat{K}(\xi, t)\right| & \leq C_{\beta} t|\xi| \log \left(e+|\xi|^{2}\right)^{\beta}|\widehat{K}(\xi, t)| \\
\left|\nabla_{\xi}^{2} \widehat{K}(\xi, t)\right| & \leq C_{\beta}\left(t \log \left(e+|\xi|^{2}\right)^{\beta}+|\xi|^{2} t^{2} \log \left(e+|\xi|^{2}\right)^{2 \beta}\right)|\widehat{K}(\xi, t)| .
\end{aligned}
$$

Then, thanks to (18) and (17), from (16), we deduce that there exists a real $\widetilde{C}_{\beta}>0$ depending only on $\beta$ such that for all $\xi \in \mathbb{R}^{2}$,

$$
\left|\nabla_{\xi}^{2}\left(\xi^{m} \widehat{K}(\xi, t)\right)\right| \leq \widetilde{C}_{\beta}\left(\delta_{m, 2}+t|\xi|^{m} \log \left(e+|\xi|^{2}\right)^{\beta}+t^{2}|\xi|^{m+2} \log \left(e+|\xi|^{2}\right)^{2 \beta}\right) e^{-t|\xi|^{2} \log \left(e+|\xi|^{2}\right)^{\beta}} .
$$

Then, using $J_{s, n}$ defined in Proposition 3.1, we deduce,

$$
\left\|\nabla_{\xi}^{2}\left(\xi^{m} \widehat{K}(\xi, t)\right)\right\|_{L^{2}}^{2} \leq 3 \widetilde{C}_{\beta}^{2}\left(\delta_{m, 2} J_{0,0}(t)+t^{2} J_{m, 2}(t)+t^{4} J_{m+2,4}(t)\right) .
$$

Thanks to Proposition 3.1, we deduce that there exists a real $\bar{C}_{\beta}>0$ depending only on $\beta$ such that for any $m \in\{0,1,2\}$,

$$
\left\|\nabla_{\xi}^{2}\left(\xi^{m} \widehat{K}(\xi, t)\right)\right\|_{L^{2}}^{2} \leq \frac{\bar{C}_{\beta}}{t^{m-1} \log \left(e+\frac{1}{t \log \left(e+\frac{1}{t}\right)^{\beta}}\right)^{\beta(m-1)}} .
$$

Thanks to (21) and Lemma 3.1 used with $s=m$, from (15) we deduce that there exists a real $Q_{\beta}>0$ depending only on $\beta$ such that,

$$
\left\|\nabla^{m} \widehat{G}(t)\right\|_{L^{1}} \leq \frac{Q_{\beta}}{t^{\frac{m}{2}} \log \left(e+\frac{1}{t \log \left(e+\frac{1}{t}\right)^{\beta}}\right)^{\frac{\beta m}{2}}} .
$$

Then, thanks to (14), we conclude the proof. 


\section{Local well-posedness of the Cauchy problem of our MHD sys- tem}

This section is devoted to the proof of our Proposition 4.3 where we establish the local well-posedness of the Cauchy problem of the partially viscous magneto-hydrodynamic system (3) with a characterization of the maximal time existence of strong solutions. To obtain these results, we begin by showing through Proposition 4.2 that the $H^{s}$-norm of $(u, b)$ is controlled by the integral in time of the maximum magnitude of the vorticity of $(u, b)$. Such a proposition has been proved in $[5,17]$ for any integer $s \geq 3$, but here we extend this result to all $s>2$. This improvement is obtained by using the logarithmic Sobolev inequality proved in $[16,15]$ which requires only that $s>2$ instead of using the one proved in [2] as it is the case in [5] and which requires that $s \geq 3$. To obtain Proposition 4.2, we need to use the following Proposition,

Proposition 4.1 Let $\left(u_{0}, b_{0}\right)$ satisfying the conditions stated in Theorem 1.1. If $(u, b) \in C\left([0, T] ; H^{s}\right)$ is the corresponding solution of (3) with $\mathfrak{D}$ given by (5), then for any $t \in[0, T]$ and for all $0 \leq r \leq s$,

$$
\|(u, b)(t)\|_{H^{r}} \leq\left\|\left(u_{0}, b_{0}\right)\right\|_{H^{r}} e^{C \int_{0}^{t}\|\nabla(u, b)(\tau)\|_{L^{\infty} d \tau}}
$$

where $C>0$ is a constant.

Proof. Applying $J^{r}$ with $J=(I-\Delta)^{\frac{1}{2}}$ to the velocity field equation and magnetic field equation, and taking the $L^{2}$ inner product of the resulting equations with $J^{r} u$ and $J^{r} b$ respectively, one has,

$$
\begin{aligned}
\frac{1}{2} \frac{d}{d t}\left(\left\|J^{r} u\right\|_{2}^{2}+\left\|J^{r} b\right\|_{2}^{2}\right)+\left\|\mathfrak{D}^{\frac{1}{2}} J^{r} b\right\|_{2}^{2} & =-\int_{\mathbb{R}^{2}} J^{r} u\left[J^{r}((u \cdot \nabla) u)-(u \cdot \nabla) J^{r} u\right] \\
& -\int_{\mathbb{R}^{2}} J^{r} b\left[J^{r}((u \cdot \nabla) b)-(u \cdot \nabla) J^{r} b\right] \\
& +\int_{\mathbb{R}^{2}} J^{r} u\left[J^{r}((b \cdot \nabla) b)-(b \cdot \nabla) J^{r} b\right] \\
& +\int_{\mathbb{R}^{2}} J^{r} b\left[J^{r}((b \cdot \nabla) u)-(b \cdot \nabla) J^{r} u\right],
\end{aligned}
$$

where we have used the divergence free condition $\nabla \cdot u=\nabla \cdot b=0$. Using the commutator estimates (1.1) in [15], we infer that there exists a constant $C>0$ such that for all $t \in[0, T]$,

$$
\frac{1}{2} \frac{d}{d t}\left(\left\|J^{r} u(t)\right\|_{2}^{2}+\left\|J^{r} b(t)\right\|_{2}^{2}\right) \lesssim\left(\|\nabla u(t)\|_{L^{\infty}}+\|\nabla b(t)\|_{L^{\infty}}\right)\left(\left\|J^{r} u(t)\right\|_{2}^{2}+\left\|J^{r} b(t)\right\|_{2}^{2}\right),
$$

which means that

$$
\frac{d}{d t}\|(u, b)(t)\|_{H^{r}} \lesssim\left(\|\nabla u(t)\|_{L^{\infty}}+\|\nabla b(t)\|_{L^{\infty}}\right)\|(u, b)(t)\|_{H^{r}} .
$$

Thus, thanks to Gronwall inequality, we infer (22), which concludes the proof.

Notice from (23) that for $r>2$ thanks to the Sobolev embedding $H^{r}\left(\mathbb{R}^{2}\right) \hookrightarrow B C^{1}\left(\mathbb{R}^{2}\right)$, we have,

$$
\frac{d}{d t}\|(u, b)(t)\|_{H^{r}} \lesssim\|(u, b)(t)\|_{H^{r}}^{2} .
$$

This estimate will be useful in establishing local existence of strong solutions.

Then, owing to Proposition 4.1, in Proposition 4.2 we give a BKM-type blow up criterion. For the proof of this latter, we use the following logarithmic Sobolev inequality which is proved in [16] (see inequality $(4.20))$ and is an improved version of that in [2]:

$$
\|\nabla f\|_{L^{\infty}\left(\mathbb{R}^{2}\right)} \lesssim 1+\|\nabla \times f\|_{L^{\infty}\left(\mathbb{R}^{2}\right)}\left(1+\log ^{+}\|f\|_{W^{s, p}\left(\mathbb{R}^{2}\right)}\right) \text { with } p>1 \text { and } s>\frac{2}{p},
$$

where $\nabla \times f=-\partial_{2} f_{1}+\partial_{1} f_{2}$ is the vorticity of $f$ and $\log ^{+} x=\max (0, \log x)$ for any $x>0$. 
Proposition 4.2 Let $\left(u_{0}, b_{0}\right)$ satisfying the conditions stated in Theorem 1.1. If $(u, b) \in C\left(\left[0, T\left[; H^{s}\right)\right.\right.$ is the corresponding solution of (3) with $u \notin C\left([0, T] ; H^{s}\right)$ and $\mathfrak{D}$ given by (5), then $\int_{0}^{T}\|(\omega, j)(t)\|_{L^{\infty}} d t=$ $\infty$, where $\omega=\nabla \times u=-\partial_{2} u_{1}+\partial_{1} u_{2}$ be the vorticity and $j=\nabla \times b=-\partial_{2} b_{1}+\partial_{1} b_{2}$ (This gives a precise meaning to the statement that $\|(u, b)\|_{H^{s}}$ does not blow up unless $\|(\omega, j)\|_{L^{\infty}}$, does $)$.

Proof. Thanks to Proposition 4.1, there exists a constant $C>0$ such that for all $t \in[0, T[$

$$
\|(u, b)(t)\|_{H^{s}} \leq\left\|\left(u_{0}, b_{0}\right)\right\|_{H^{s}} e^{C \int_{0}^{t}\|\nabla(u, b)(\tau)\|_{L} \infty d \tau} .
$$

Thanks to (25) used with $p=2$, from (26) we deduce that there exists a constant $C_{1}>1$ such that for all $t \in[0, T[$

$$
\|(u, b)(t)\|_{H^{s}} \leq C_{1}\left\|\left(u_{0}, b_{0}\right)\right\|_{H^{s}} e^{C_{1} \int_{0}^{t}\|(\omega, j)(\tau)\|_{L} \infty\left(1+\log ^{+}\|(u, b)(\tau)\|_{H^{s}}\right) d \tau} .
$$

Therefore by applying the function $\log ^{+}$to $(27)$, we deduce that for all $t \in[0, T[$,

$$
\log ^{+}\|(u, b)(t)\|_{H^{s}} \leq \log ^{+}\left(C_{1}\left\|\left(u_{0}, b_{0}\right)\right\|_{H^{s}}\right)+C_{1} \int_{0}^{t}\|(\omega, j)(\tau)\|_{L^{\infty}}\left(1+\log ^{+}\|(u, b)(\tau)\|_{H^{s}}\right) d \tau,
$$

which yields to

$$
1+\log ^{+}\|(u, b)(t)\|_{H^{s}} \leq 1+\log ^{+}\left(C_{1}\left\|\left(u_{0}, b_{0}\right)\right\|_{H^{s}}\right)+C_{1} \int_{0}^{t}\|(\omega, j)(\tau)\|_{L^{\infty}}\left(1+\log ^{+}\|(u, b)(\tau)\|_{H^{s}}\right) d \tau \text {. }
$$

Then from (29), thanks to Gronwall Lemma, we deduce that for all $t \in[0, T[$,

$$
1+\log ^{+}\|(u, b)(t)\|_{H^{s}} \leq\left(1+\log ^{+}\left(C_{1}\left\|\left(u_{0}, b_{0}\right)\right\|_{H^{s}}\right)\right) e^{C_{1} \int_{0}^{t}\|(\omega, j)(\tau)\|_{L} \infty d \tau} .
$$

Then plugging (30) into (27) yields to that for all $t \in[0, T[$,

$$
\begin{aligned}
\|(u, b)(t)\|_{H^{s}} & \leq C_{1}\left\|\left(u_{0}, b_{0}\right)\right\|_{H^{s}} e^{\left(1+\log ^{+}\left(C_{1}\left\|\left(u_{0}, b_{0}\right)\right\|_{H^{s}}\right)\right) \int_{0}^{t} C_{1}\|(\omega, j)(\tau)\|_{L^{\infty}} e^{C_{1} \int_{0}^{\tau}\|(\omega, j)(s)\|_{L} \infty d s} d \tau} \\
& =C_{1}\left\|\left(u_{0}, b_{0}\right)\right\|_{H^{s}} \exp \left(\left(1+\log ^{+}\left(C_{1}\left\|\left(u_{0}, b_{0}\right)\right\|_{H^{s}}\right)\right)\left(e^{C_{1} \int_{0}^{t}\|(\omega, j)(\tau)\|_{L} \infty d \tau}-1\right)\right) \\
& \leq C_{1}\left\|\left(u_{0}, b_{0}\right)\right\|_{H^{s}} \exp \left(\left(1+\log ^{+}\left(C_{1}\left\|\left(u_{0}, b_{0}\right)\right\|_{H^{s}}\right)\right) e^{C_{1} \int_{0}^{t}\|(\omega, j)(\tau)\|_{L} \infty d \tau}\right) .
\end{aligned}
$$

Then, from this last inequality, we conclude the proof.

Before to turn of the proof of Proposition 4.3, we provide two simple bounds. The first one is a $L^{2}$-energy estimate given in Lemma 4.1 and the second one is a $H^{1}$-energy estimate given in Lemma 4.2.

Multiplying the first two equations of (3) by $u$ and $b$, respectively, integrating and adding the resulting equations together, Lemma 4.1 follows.

Lemma 4.1 Let $\left(u_{0}, b_{0}\right)$ satisfying the conditions stated in Theorem 1.1. If $(u, b) \in C\left([0, T] ; H^{s}\right)$ is the corresponding solution of (3) and $\mathfrak{D}$ given by (5), then, for any $t \in[0, T]$,

$$
\|u(t)\|_{2}^{2}+\|b(t)\|_{2}^{2}+2 \int_{0}^{t}\left\|\mathfrak{D}^{\frac{1}{2}} b(\tau)\right\|_{2}^{2} d \tau=\left\|u_{0}\right\|_{2}^{2}+\left\|b_{0}\right\|_{2}^{2} .
$$

Let $\omega=\nabla \times u=-\partial_{2} u_{1}+\partial_{1} u_{2}$ be the vorticity and $j=\nabla \times b=-\partial_{2} b_{1}+\partial_{1} b_{2}$ be the current density. Applying $\nabla \times$ the first two equations of (3) we obtain the governing equations.

$$
\left\{\begin{array}{l}
\partial_{t} \omega+(u \cdot \nabla) \omega=(b \cdot \nabla) j \\
\partial_{t} j+(u \cdot \nabla) j=(b \cdot \nabla) \omega+T(\nabla u, \nabla b)-\mathfrak{D} j .
\end{array}\right.
$$

where,

$$
T(\nabla u, \nabla b)=2 \partial_{1} b_{1}\left(\partial_{2} u_{1}+\partial_{1} u_{2}\right)+2 \partial_{2} u_{2}\left(\partial_{2} b_{1}+\partial_{1} b_{2}\right) .
$$

Then, we get a $H^{1}$-bound on $(u, b)$ obtained as in [20, 17], namely we have Lemma 4.2. 
Lemma 4.2 Let $\left(u_{0}, b_{0}\right)$ satisfying the conditions stated in Theorem 1.1. If $(u, b) \in C\left([0, T] ; H^{s}\right)$ is the corresponding solution of (3) and $\mathfrak{D}$ given by (5), then, there exists a constant $C>0$ such that for any $t \in[0, T]$,

$$
\|\omega(t)\|_{2}^{2}+\|j(t)\|_{2}^{2}+\int_{0}^{t}\left\|\mathfrak{D}^{\frac{1}{2}} j(\tau)\right\|_{2}^{2} d \tau \leq\left(\left\|\omega_{0}\right\|_{2}^{2}+\left\|j_{0}\right\|_{2}^{2}\right) e^{C\left(\left\|u_{0}\right\|_{2}^{2}+\left\|b_{0}\right\|_{2}^{2}\right)} .
$$

Proof. Taking the $L^{2}$ inner product of the equations (31) with $\omega$ and $j$ respectively and adding the resulting equations together, one has for all $t \in[0, T]$

$$
\frac{1}{2} \frac{d}{d t}\left(\|\omega\|_{2}^{2}+\|j\|_{2}^{2}\right)+\left\|\mathfrak{D}^{\frac{1}{2}} j\right\|_{2}^{2}=\int_{\mathbb{R}^{2}} T(\nabla u, \nabla b) j
$$

where we have used the following consequences of $\nabla \cdot u=0$ and $\nabla \cdot b=0$ :

$$
\int_{\mathbb{R}^{2}} u \cdot \nabla \omega \omega=\int_{\mathbb{R}^{2}} u \cdot \nabla\left(\frac{\omega^{2}}{2}\right)=-\int_{\mathbb{R}^{2}}(\nabla \cdot u) \frac{\omega^{2}}{2}=0
$$

and similarly $\int_{\mathbb{R}^{2}} u \cdot \nabla j j=0$ and we get also

$$
\int_{\mathbb{R}^{2}} b \cdot \nabla j \omega+\int_{\mathbb{R}^{2}} b \cdot \nabla \omega j=\int_{\mathbb{R}^{2}} b \cdot \nabla(j \omega)=-\int_{\mathbb{R}^{2}}(\nabla \cdot b) j \omega=0 .
$$

Then, using Cauchy-Schwarz inequality and Hölder inequality, from (32), we obtain,

$$
\begin{aligned}
\frac{1}{2} \frac{d}{d t}\left(\|\omega\|_{2}^{2}+\|j\|_{2}^{2}\right)+\left\|\mathfrak{D}^{\frac{1}{2}} j\right\|_{2}^{2} & \lesssim\|\nabla u\|_{2}\|j \nabla b\|_{2} \\
& \leq\|\nabla u\|_{2}\|j\|_{4}\|\nabla b\|_{4} .
\end{aligned}
$$

Thanks to Calderon-Zygmund theory (see Theorem 3.1.1 in [4]), we get,

$$
\|\nabla u\|_{2} \lesssim\|\omega\|_{2} \text { and }\|\nabla b\|_{4} \lesssim\|j\|_{4}
$$

From (33), we deduce,

$$
\frac{1}{2} \frac{d}{d t}\left(\|\omega\|_{2}^{2}+\|j\|_{2}^{2}\right)+\left\|\mathfrak{D}^{\frac{1}{2}} j\right\|_{2}^{2} \lesssim\|\omega\|_{2}\|j\|_{4}^{2} .
$$

Next, application to the Gagliardo-Nirenberg inequality,

$$
\|j\|_{4} \lesssim\|j\|_{2}^{\frac{1}{2}}\|\nabla j\|_{2}^{\frac{1}{2}}
$$

yields from (34), that there exists a constant $C>0$ such that

$$
\frac{1}{2} \frac{d}{d t}\left(\|\omega\|_{2}^{2}+\|j\|_{2}^{2}\right)+\left\|\mathfrak{D}^{\frac{1}{2}} j\right\|_{2}^{2} \leq C\|\omega\|_{2}\|j\|_{2}\|\nabla j\|_{2} .
$$

By using Plancherel identity, we observe that $\left\|\mathfrak{D}^{\frac{1}{2}} j\right\|_{2} \geq\|\nabla j\|_{2}$. Then thanks to Young inequality, from (35) we infer

which yields to

$$
\frac{1}{2} \frac{d}{d t}\left(\|\omega\|_{2}^{2}+\|j\|_{2}^{2}\right)+\frac{1}{2}\left\|\mathfrak{D}^{\frac{1}{2}} j\right\|_{2}^{2} \leq \frac{C^{2}}{2}\|\omega\|_{2}^{2}\|j\|_{2}^{2},
$$

$$
\frac{d}{d t}\left(\|\omega\|_{2}^{2}+\|j\|_{2}^{2}\right) \leq C^{2}\left(\|\omega\|_{2}^{2}+\|j\|_{2}^{2}\right)\|j\|_{2}^{2}
$$

Then from (37), thanks to Gronwall Lemma, we deduce that for all $t \in[0, T]$

$$
\|\omega(t)\|_{2}^{2}+\|j(t)\|_{2}^{2} \leq\left(\left\|\omega_{0}\right\|_{2}^{2}+\left\|j_{0}\right\|_{2}^{2}\right) e^{C^{2} \int_{0}^{t}\|j(\tau)\|_{2}^{2} d \tau} .
$$


By using (38) to bound $\|\omega\|_{2}^{2}$ at the right hand side of inequality (36), we get that for all $t \in[0, T]$,

$$
\frac{d}{d t}\left(\|\omega(t)\|_{2}^{2}+\|j(t)\|_{2}^{2}\right)+\left\|\mathfrak{D}^{\frac{1}{2}} j(t)\right\|_{2}^{2} \leq\left(\left\|\omega_{0}\right\|_{2}^{2}+\left\|j_{0}\right\|_{2}^{2}\right) C^{2}\|j(t)\|_{2}^{2} e^{C^{2} \int_{0}^{t}\|j(\tau)\|_{2}^{2} d \tau} .
$$

Integrating inequality (39) over $[0, t]$, we infer that for all $t \in[0, T]$,

$$
\|\omega(t)\|_{2}^{2}+\|j(t)\|_{2}^{2}+\int_{0}^{t}\left\|\mathfrak{D}^{\frac{1}{2}} j(\tau)\right\|_{2}^{2} d \tau \leq\left(\left\|\omega_{0}\right\|_{2}^{2}+\left\|j_{0}\right\|_{2}^{2}\right) e^{C^{2} \int_{0}^{t}\|j(\tau)\|_{2}^{2} d \tau} .
$$

Since $\|j(\tau)\|_{2} \leq 2\|\nabla b(\tau)\|_{2} \leq 2\left\|\mathfrak{D}^{\frac{1}{2}} b(\tau)\right\|_{2}$ and thanks to Lemma 4.1, from (40) we obtain that for all $t \in[0, T]$

$$
\|\omega(t)\|_{2}^{2}+\|j(t)\|_{2}^{2}+\int_{0}^{t}\left\|\mathfrak{D}^{\frac{1}{2}} j(\tau)\right\|_{2}^{2} d \tau \leq\left(\left\|\omega_{0}\right\|_{2}^{2}+\left\|j_{0}\right\|_{2}^{2}\right) e^{\frac{C^{2}}{2}\left(\left\|u_{0}\right\|_{2}^{2}+\left\|b_{0}\right\|_{2}^{2}\right)},
$$

which concludes the proof.

Now, we can give the proof of Proposition 4.3.

Proposition 4.3 Assume that $\left(u_{0}, b_{0}\right) \in H^{s}\left(\mathbb{R}^{2}\right)$ with $s>2, \nabla \cdot u_{0}=0, \nabla \cdot b_{0}=0$. Then there exists a maximal time of existence $T^{*}>0$ such that there exists a unique solution $(u, b) \in C\left(\left[0, T^{*}\left[; H^{s}\left(\mathbb{R}^{2}\right)\right)\right.\right.$ of the system of Equations (3) with $\mathfrak{D}$ given by (5).

Moreover if $T^{*}<\infty$, then

$$
\int_{0}^{T^{*}}\|(\omega, j)(\tau)\|_{L^{\infty}} d \tau=\infty
$$

where $\omega=\nabla \times u=-\partial_{2} u_{1}+\partial_{1} u_{2}$ is the vorticity and $j=\nabla \times b=-\partial_{2} b_{1}+\partial_{1} b_{2}$.

Proof. The first part of the proof concerning only the local existence of solutions in $C\left([0, T] ; H^{s}\left(\mathbb{R}^{2}\right)\right)$ for some $T>0$ follows from the arguments used at pp. 20 of [19], by implementing a Galerkin method using as a basis of $H:=\mathbb{P} L^{2}\left(\mathbb{R}^{2}\right)$ the eigenfunctions of the operator $\mathfrak{D}$, using the a-priori estimates obtained in Lemmata 4.1, 4.2 and the a-priori inequality obtained in (24). Uniqueness is then obtained by using the same arguments as in Proposition 4.1. Then we deduce that there exists a maximal time of existence $T^{*}>0$ such that there exists a unique solution $(u, b) \in C\left(\left[0, T^{*}\left[; H^{s}\left(\mathbb{R}^{2}\right)\right)\right.\right.$ of the system of Equations (3) with $\mathfrak{D}$ given by (5). Thanks to Proposition 4.2, we deduce that if $T^{*}<\infty$, then

$$
\int_{0}^{T^{*}}\|(\omega, j)(\tau)\|_{L^{\infty}} d \tau=\infty
$$

which completes the proof.

\section{Global regularity}

In this section, we prove our Theorem 1.1. To get our Theorem, we need to prove a series of Lemmata 5.1, 5.2 and 5.3. We begin from the following Lemma obtained from Lemmata 4.1 and 4.2.

Lemma 5.1 Let $\left(u_{0}, b_{0}\right)$ satisfying the conditions stated in Theorem 1.1. If $(u, b) \in C\left(\left[0, T\left[; H^{s}\right)\right.\right.$ is the unique corresponding solution of (3) and $\mathfrak{D}$ given by (5), then, there exists a real $C>0$ depending continuously only on $\beta,\left\|b_{0}\right\|_{L^{\infty}},\left\|u_{0}\right\|_{2},\left\|b_{0}\right\|_{2},\left\|\omega_{0}\right\|_{2},\left\|j_{0}\right\|_{2}, T$ such that,

$$
\|b(t)\|_{L^{\infty}\left(\mathbb{R}^{2} \times[0, T]\right)} \leq C .
$$


Proof. We write the second equation (3) under its integral form, thanks to the kernel $K$ given in Section 3 , we have for all $t \in[0, T[$,

$$
b(t)=K(t) \star b_{0}+\int_{0}^{t} K(t-\tau) \star((b(\tau) \cdot \nabla) u(\tau)-(u(\tau) \cdot \nabla) b(\tau)) d \tau .
$$

Since $\nabla \cdot b(\tau)=\nabla \cdot u(\tau)=0$, we have $(b(\tau) \cdot \nabla) u(\tau)=\nabla \cdot(b(\tau) \otimes u(\tau))$ and $(u(\tau) \cdot \nabla) b(\tau)=\nabla \cdot(u(\tau) \otimes b(\tau))$, then taking the $L^{\infty}$-norm in equation (42) and using Young inequality, we deduce for all $t \in[0, T$,

$$
\begin{aligned}
\|b(t)\|_{L^{\infty}} & \leq\left\|K(t) \star b_{0}\right\|_{L^{\infty}}+\int_{0}^{t}\|\nabla K(t-\tau)\|_{L^{2}}\|u(\tau) b(\tau)\|_{2} d \tau \\
& \leq\|K(t)\|_{L^{1}}\left\|b_{0}\right\|_{L^{\infty}}+\int_{0}^{t}\|\nabla K(t-\tau)\|_{L^{2}}\|u(\tau)\|_{4}\|b(\tau)\|_{4} d \tau .
\end{aligned}
$$

Thanks to Lemma 3.3, there exists a real $C_{0}>0$ depending only on $\beta$ such that $\|K(t)\|_{L^{1}} \leq C_{0}$. Thanks to Gagliardo-Nirenberg inequality, we have, $\|u(\tau)\|_{4} \lesssim\|u(\tau)\|_{2}^{\frac{1}{2}}\|\nabla u(\tau)\|_{2}^{\frac{1}{2}}$ and thanks to Theorem 3.1.1 in [4], we have $\|\nabla u(\tau)\|_{2} \lesssim\|\omega(\tau)\|_{2}$, hence we get $\|u(\tau)\|_{4} \lesssim\|u(\tau)\|_{2}^{\frac{1}{2}}\|\omega(\tau)\|_{2}^{\frac{1}{2}}$, similarly $\|b(\tau)\|_{4} \lesssim$ $\|b(\tau)\|_{2}^{\frac{1}{2}}\|j(\tau)\|_{2}^{\frac{1}{2}}$. Then, thanks to Lemmata 4.1 and 4.2 , we deduce that there exists a real $C>0$ depending only on $\left\|u_{0}\right\|_{2},\left\|b_{0}\right\|_{2},\left\|\omega_{0}\right\|_{2},\left\|j_{0}\right\|_{2}$ such that for all $t \in[0, T[$,

$$
\begin{aligned}
\|b(t)\|_{L^{\infty}} & \leq C_{0}\left\|b_{0}\right\|_{L^{\infty}}+C \int_{0}^{t}\|\nabla K(t-\tau)\|_{L^{2}} d \tau \\
& =C_{0}\left\|b_{0}\right\|_{L^{\infty}}+C \int_{0}^{t}\|\nabla K(\sigma)\|_{L^{2}} d \sigma .
\end{aligned}
$$

Thanks to Lemma 3.1 used with $s=1$ and since $\beta>1$, we deduce for any $t \geq 0, \int_{0}^{t}\|\nabla K(\sigma)\|_{L^{2}} d \sigma<+\infty$, combined with (44), we conclude the proof.

Lemma 5.2 Let $\left(u_{0}, b_{0}\right)$ satisfying the conditions stated in Theorem 1.1. If $(u, b) \in C\left(\left[0, T\left[; H^{s}\right)\right.\right.$ is the unique corresponding solution of (3) and $\mathfrak{D}$ given by (5), then there exists a real $C>0$ depending continuously only on $\beta,\left\|u_{0}\right\|_{2},\left\|b_{0}\right\|_{2},\left\|\omega_{0}\right\|_{2},\left\|j_{0}\right\|_{2},\left\|b_{0}\right\|_{L^{\infty}}, T$ such that,

$$
\int_{0}^{T}\|j(\tau)\|_{L^{\infty}} d \tau \leq C
$$

Proof. We write the second equation (31) under its integral form, thanks to the kernel $K$ given in Section 3 , we have for all $t \in[0, T[$,

$$
j(t)=K(t) \star j_{0}+\int_{0}^{t} K(t-\sigma) \star((b(\sigma) \cdot \nabla) \omega(\sigma)-(u(\sigma) \cdot \nabla) j(\sigma)+T(\nabla u(\sigma), \nabla b(\sigma))) d \sigma .
$$

Since $\nabla \cdot b(\sigma)=\nabla \cdot u(\sigma)=0$, we have $(b(\sigma) \cdot \nabla) \omega(\sigma)=\nabla \cdot(b(\sigma) \otimes \omega(\sigma))$ and $(u(\sigma) \cdot \nabla) j(\sigma)=\nabla \cdot(u(\sigma) \otimes j(\sigma))$, then taking the $L^{\infty}$-norm in equation (45) and using Young inequality, we deduce,

$$
\begin{aligned}
\|j(t)\|_{L^{\infty}} & \leq\|K(t)\|_{2}\left\|j_{0}\right\|_{2}+\int_{0}^{t}\|\nabla K(t-\sigma)\|_{L^{2}}\left(\|b(\sigma) \otimes \omega(\sigma)\|_{2}+\|u(\sigma) \otimes j(\sigma)\|_{2}\right) d \sigma \\
& +\int_{0}^{t}\|K(t-\sigma)\|_{L^{\infty}}\|T(\nabla u(\sigma), \nabla b(\sigma))\|_{1} d \sigma .
\end{aligned}
$$

We observe,

$$
\|b(\sigma) \otimes \omega(\sigma)\|_{2} \leq\|b(\sigma)\|_{L^{\infty}}\|\omega(\sigma)\|_{2}
$$


Thanks to Cauchy-Schwarz inequality, Gagliardo-Nirenberg inequality and Young inequality, we get,

$$
\begin{aligned}
\|u(\sigma) \otimes j(\sigma)\|_{2} & \leq\|u(\sigma)\|_{4}\|j(\sigma)\|_{4} \\
& \lesssim\|u(\sigma)\|_{2}^{\frac{1}{2}}\|\nabla u(\sigma)\|_{2}^{\frac{1}{2}}\|j(\sigma)\|_{2}^{\frac{1}{2}}\|\nabla j(\sigma)\|_{2}^{\frac{1}{2}} \\
& \lesssim\|\nabla u(\sigma)\|_{2}\|j(\sigma)\|_{2}+\|u(\sigma)\|_{2}\|\nabla j(\sigma)\|_{2} \\
& \lesssim\|\omega(\sigma)\|_{2}\|j(\sigma)\|_{2}+\|u(\sigma)\|_{2}\|\nabla j(\sigma)\|_{2},
\end{aligned}
$$

where we have used the fact that $\|\nabla u(\sigma)\|_{2} \lesssim\|\omega(\sigma)\|_{2}$, thanks to Theorem 3.1.1 in [4]. Thanks to Cauchy-Schwarz inequality, we have,

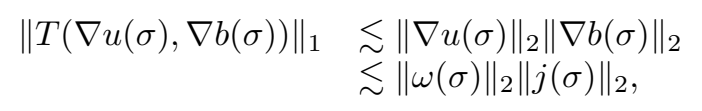

where we have used Theorem 3.1.1 in [4]. Thanks to Lemmata 4.1, 4.2 and 5.1 used in the inequalities (47), (48) and (49), from (46), we deduce that there exists a real $C>0$ depending only on $\beta,\left\|u_{0}\right\|_{2},\left\|b_{0}\right\|_{2},\left\|\omega_{0}\right\|_{2},\left\|j_{0}\right\|_{2},\left\|b_{0}\right\|_{L^{\infty}}$ such that,

$$
\|j(t)\|_{L^{\infty}} \leq C\left(\|K(t)\|_{2}+\int_{0}^{t}\|\nabla K(t-\sigma)\|_{L^{2}} d \sigma+\int_{0}^{t}\|K(t-\sigma)\|_{L^{\infty}} d \sigma+\int_{0}^{t}\|\nabla K(t-\sigma)\|_{L^{2}}\|\nabla j(\sigma)\|_{2} d \sigma\right) .
$$

Thanks to Lemma 3.1 used with $s=0$, we deduce that for any $t \in\left[0, T\left[,\|K(t)\|_{2} \lesssim \frac{1}{\sqrt{t}}\right.\right.$. Thanks again to Lemma 3.1 used with $s=1$, Lemma 3.2 and since $\beta>1$, we observe that for any $t \geq 0, g(t):=$ $\int_{0}^{t}\|\nabla K(\sigma)\|_{L^{2}} d \sigma<+\infty$ and $h(t):=\int_{0}^{t}\|K(\sigma)\|_{L^{\infty}} d \sigma<+\infty$. Notice that $g$ and $h$ are continuous non-decreasing function. We re-write Inequality (50) as follows, for any $\tau \in] 0, T[$,

$$
\|j(\tau)\|_{L^{\infty}} \lesssim C\left(\frac{1}{\sqrt{\tau}}+g(\tau)+h(\tau)+\int_{0}^{\tau}\|\nabla K(\tau-\sigma)\|_{L^{2}}\|\nabla j(\sigma)\|_{2} d \sigma\right) .
$$

We integrate Inequality (51) over $\tau \in] 0, t]$, to obtain for all $t \in[0, T[$,

$$
\int_{0}^{t}\|j(\tau)\|_{L^{\infty}} d \tau \lesssim C\left(2 \sqrt{t}+t(g(t)+h(t))+\int_{0}^{t} \int_{0}^{\tau}\|\nabla K(\tau-\sigma)\|_{L^{2}}\|\nabla j(\sigma)\|_{2} d \sigma d \tau\right) .
$$

By inverting the integrals, we deduce,

$$
\begin{aligned}
\int_{0}^{t} \int_{0}^{\tau}\|\nabla K(\tau-\sigma)\|_{L^{2}}\|\nabla j(\sigma)\|_{2} d \sigma d \tau & =\int_{0}^{t}\|\nabla j(\sigma)\|_{2}\left(\int_{\sigma}^{t}\|\nabla K(\tau-\sigma)\|_{L^{2}} d \tau\right) d \sigma \\
& =\int_{0}^{t}\|\nabla j(\sigma)\|_{2} g(t-\sigma) d \sigma .
\end{aligned}
$$

Since $g$ is a non-decreasing function, we deduce,

$$
\begin{aligned}
\int_{0}^{t} \int_{0}^{\tau}\|\nabla K(\tau-\sigma)\|_{L^{2}}\|\nabla j(\sigma)\|_{2} d \sigma d \tau & \leq g(t) \int_{0}^{t}\|\nabla j(\sigma)\|_{2} d \sigma \\
& \leq g(t) \sqrt{t}\left(\int_{0}^{t}\|\nabla j(\sigma)\|_{2}^{2} d \sigma\right)^{\frac{1}{2}}
\end{aligned}
$$

where we have used Cauchy-Schwarz inequality. Thanks to Lemma 4.2, we deduce that there exists a constant $\widetilde{C}>0$ such that,

$$
2 \int_{0}^{t}\|\nabla j(\sigma)\|_{2}^{2} d \sigma \leq\left(\left\|\omega_{0}\right\|_{2}^{2}+\left\|j_{0}\right\|_{2}^{2}\right) e^{\widetilde{C}\left(\left\|u_{0}\right\|_{2}^{2}+\left\|b_{0}\right\|_{2}^{2}\right)} .
$$


Thanks to (53), (54), from (52), we deduce that there exists a continuous non-decreasing function $f$ on $\mathbb{R}_{+}$ depending only on $\beta,\left\|u_{0}\right\|_{2},\left\|b_{0}\right\|_{2},\left\|\omega_{0}\right\|_{2},\left\|j_{0}\right\|_{2},\left\|b_{0}\right\|_{L^{\infty}}$ such that for all $t \in\left[0, T\left[, \int_{0}^{t}\|j(\tau)\|_{L^{\infty}} d \tau \leq f(t)\right.\right.$ and thus we infer that $\int_{0}^{T}\|j(\tau)\|_{L^{\infty}} \leq f(T)<\infty$, which concludes the proof.

Lemma 5.3 Let $\left(u_{0}, b_{0}\right)$ satisfying the conditions stated in Theorem 1.1. If $(u, b) \in C\left(\left[0, T\left[; H^{s}\right)\right.\right.$ is the unique corresponding solution of (3) and $\mathfrak{D}$ given by (5), then there exists a real $C_{0}>0$ depending continuously only on $\beta,\left\|\omega_{0}\right\|_{L^{\infty}},\left\|b_{0}\right\|_{L^{\infty}},\left\|u_{0}\right\|_{2},\left\|b_{0}\right\|_{2},\left\|\omega_{0}\right\|_{2},\left\|j_{0}\right\|_{2}, T$ such that,

$$
\int_{0}^{T}\|\nabla j(\tau)\|_{L^{\infty}} d \tau \leq C_{0}
$$

Moreover, we have that there exists a real $C_{1}>0$ depending continuously only on $\beta,\left\|\omega_{0}\right\|_{L^{\infty}},\left\|b_{0}\right\|_{L^{\infty}}$, $\left\|u_{0}\right\|_{2},\left\|b_{0}\right\|_{2},\left\|\omega_{0}\right\|_{2},\left\|j_{0}\right\|_{2}, T$ such that,

$$
\|\omega\|_{L^{\infty}\left(\mathbb{R}^{2} \times[0, T]\right)} \leq C_{1} .
$$

Proof. We write the second equation (31) under its integral form, thanks to the kernel $K$ given in Section 3 , for all $\sigma \in[0, T[$,

$$
j(\sigma)=K(\sigma) \star j_{0}+\int_{0}^{\sigma} K(\sigma-\tau) \star((b(\tau) \cdot \nabla) \omega(\tau)-(u(\tau) \cdot \nabla) j(\tau)+T(\nabla u(\tau), \nabla b(\tau))) d \tau .
$$

Since $\nabla \cdot b(\tau)=\nabla \cdot u(\tau)=0$, we have $(b(\tau) \cdot \nabla) \omega(\tau)=\nabla \cdot(b(\tau) \otimes \omega(\tau))$ and $(u(\tau) \cdot \nabla) j(\tau)=\nabla \cdot(u(\tau) \otimes j(\tau))$, then taking the operator $\nabla$, the $L^{\infty}$-norm in equation (57) and using Young inequality, we deduce that for all $\sigma \in] 0, T[$,

$$
\begin{aligned}
\|\nabla j(\sigma)\|_{L^{\infty}} & \leq\|\nabla K(\sigma)\|_{2}\left\|j_{0}\right\|_{2}+\int_{0}^{\sigma}\left\|\nabla^{2} K(\sigma-\tau)\right\|_{L^{1}}\left(\|b(\tau) \otimes \omega(\tau)\|_{L^{\infty}}+\|u(\tau) \otimes j(\tau)\|_{L^{\infty}}\right) d \tau \\
& +\int_{0}^{\sigma}\|\nabla K(\sigma-\tau)\|_{L^{2}}\|T(\nabla u(\tau), \nabla b(\tau))\|_{2} d \tau
\end{aligned}
$$

Thanks to Lemma 3.3 used with $m=2$, Lemma 3.1 used with $s=1$ and since $\beta>1$, we observe that for all $t \geq 0, r(t):=\int_{0}^{t}\left\|\nabla^{2} K(\sigma)\right\|_{L^{1}} d \sigma<+\infty$ and $g(t):=\int_{0}^{t}\|\nabla K(\sigma)\|_{L^{2}} d \sigma<+\infty$.

We integrate Inequality (58) over $\sigma \in[0, t]$ with $t \in] 0, T[$ and after inverting the integrals, we obtain,

$$
\begin{aligned}
\int_{0}^{t}\|\nabla j(\sigma)\|_{L^{\infty}} d \sigma & \leq g(t)\left\|j_{0}\right\|_{2}+\int_{0}^{t} r(t-\tau)\left(\|b(\tau) \otimes \omega(\tau)\|_{L^{\infty}}+\|u(\tau) \otimes j(\tau)\|_{L^{\infty}}\right) d \tau \\
& +\int_{0}^{t} g(t-\tau)\|T(\nabla u(\tau), \nabla b(\tau))\|_{2} d \tau
\end{aligned}
$$

Then, we deduce,

$$
\begin{aligned}
\int_{0}^{t}\|\nabla j(\sigma)\|_{L^{\infty}} d \sigma & \leq g(t)\left\|j_{0}\right\|_{2}+r(t) \int_{0}^{t}\left(\|b(\tau) \otimes \omega(\tau)\|_{L^{\infty}}+\|u(\tau) \otimes j(\tau)\|_{L^{\infty}}\right) d \tau \\
& +g(t) \int_{0}^{t}\|T(\nabla u(\tau), \nabla b(\tau))\|_{2} d \tau .
\end{aligned}
$$

By following step by step the proof of Lemma 4 given in [9] but keeping the term $\|u\|_{L^{2}\left(\mathbb{R}^{2}\right)}$ which appears after using the Cauchy-Schwarz inequality, we obtain,

$$
\begin{aligned}
\|u(\tau)\|_{L^{\infty}} & \lesssim\|u(\tau)\|_{2}^{\frac{1}{2}}\|\omega(\tau)\|_{L^{\infty}}^{\frac{1}{2}} \\
& \leq\left(\left\|u_{0}\right\|_{2}^{2}+\left\|b_{0}\right\|_{2}^{2}\right)^{\frac{1}{4}}\|\omega(\tau)\|_{L^{\infty}}^{\frac{1}{2}} \\
& \leq\left(\left\|u_{0}\right\|_{2}^{2}+\left\|b_{0}\right\|_{2}^{2}\right)^{\frac{1}{2}}+\|\omega(\tau)\|_{L^{\infty}}
\end{aligned}
$$


where we have used Lemma 4.1 and Young inequality.

Thanks to Cauchy-Schwarz inequality, Theorem 3.1.1 in [4], Interpolation inequality and Young inequality, we get,

$$
\begin{aligned}
\|T(\nabla u(\tau), \nabla b(\tau))\|_{2} & \lesssim\|\nabla u(\tau)\|_{4}\|\nabla b(\tau)\|_{4} \\
& \lesssim\|\omega(\tau)\|_{4}\|j(\tau)\|_{4} \\
& \leq\|\omega(\tau)\|_{2}^{\frac{1}{2}}\|\omega(\tau)\|_{L^{\infty}}^{\frac{1}{2}}\|j(\tau)\|_{2}^{\frac{1}{2}}\|j(\tau)\|_{L^{\infty}}^{\frac{1}{2}} \\
& \leq\|\omega(\tau)\|_{L^{\infty}}\|j(\tau)\|_{L^{\infty}}+\|\omega(\tau)\|_{2}\|j(\tau)\|_{2} .
\end{aligned}
$$

Thanks to Lemma 4.2, we deduce that there exists a real $C_{2}>0$ depending only on $\left\|u_{0}\right\|_{2},\left\|b_{0}\right\|_{2},\left\|\omega_{0}\right\|_{2},\left\|j_{0}\right\|_{2}$ such that,

$$
\|T(\nabla u(\tau), \nabla b(\tau))\|_{2} \lesssim\|\omega(\tau)\|_{L^{\infty}}\|j(\tau)\|_{L^{\infty}}+C_{2} .
$$

Therefore, thanks to (61) and (63), from (60), we deduce,

$$
\begin{aligned}
\int_{0}^{t}\|\nabla j(\sigma)\|_{L^{\infty}} d \sigma & \lesssim g(t)\left\|j_{0}\right\|_{2}+r(t)\left(\left\|u_{0}\right\|_{2}^{2}+\left\|b_{0}\right\|_{2}^{2}\right)^{\frac{1}{2}} \int_{0}^{t}\|j(\tau)\|_{L^{\infty}} d \tau+g(t) t C_{2} \\
& +r(t) \int_{0}^{t}\|b(\tau)\|_{L^{\infty}}\|\omega(\tau)\|_{L^{\infty}} d \tau+(g(t)+r(t)) \int_{0}^{t}\|j(\tau)\|_{L^{\infty}}\|\omega(\tau)\|_{L^{\infty}} d \tau .
\end{aligned}
$$

Let us estimate $\|\omega(\tau)\|_{L^{\infty}}$. Let $2 \leq p<\infty$. Multiplying the first equation in (31) by $\omega|\omega|^{p-2}$, integrating in space and applying Hölder's inequality, we have for any $t \in[0, T[$,

$$
\frac{1}{p} \frac{d}{d t}\|\omega(t)\|_{p}^{p}=\int b(t) \cdot \nabla j(t) \omega(t)|\omega(t)|^{p-2} \leq\|b(t)\|_{L^{\infty}}\|\omega(t)\|_{p}^{p-1}\|\nabla j(t)\|_{p},
$$

which yields to,

$$
\frac{d}{d t}\|\omega(t)\|_{p} \leq\|b(t)\|_{L^{\infty}}\|\nabla j(t)\|_{p}
$$

Then, from (65) we deduce,

$$
\|\omega(t)\|_{p} \leq\left\|\omega_{0}\right\|_{p}+\int_{0}^{t}\|b(\tau)\|_{L^{\infty}}\|\nabla j(\tau)\|_{p} d \tau .
$$

Letting $p \rightarrow \infty$ in (66), we infer that for all $\tau \in[0, T[$,

$$
\|\omega(\tau)\|_{L^{\infty}} \leq\left\|\omega_{0}\right\|_{L^{\infty}}+\int_{0}^{\tau}\|b(\sigma)\|_{L^{\infty}}\|\nabla j(\sigma)\|_{L^{\infty}} d \sigma .
$$

Plugging inequality (67) into (64), thanks to Lemma 5.1 and Lemma 5.2, we deduce that there exists real $A>0, B>0$ and $C>0$ depending only on $\beta,\left\|\omega_{0}\right\|_{L^{\infty}},\left\|b_{0}\right\|_{L^{\infty}},\left\|u_{0}\right\|_{2},\left\|b_{0}\right\|_{2},\left\|\omega_{0}\right\|_{2},\left\|j_{0}\right\|_{2}, T$ such that for all $t \in[0, T[$,

$$
\int_{0}^{t}\|\nabla j(\sigma)\|_{L^{\infty}} d \sigma \leq A+\int_{0}^{t}\left(B+C\|j(\tau)\|_{L^{\infty}}\right)\left(\int_{0}^{\tau}\|\nabla j(\sigma)\|_{L^{\infty}} d \sigma\right) d \tau .
$$

Thanks to Gronwall inequality, we deduce that for all $t \in[0, T[$,

$$
\int_{0}^{t}\|\nabla j(\sigma)\|_{L^{\infty}} d \sigma \quad \leq A \exp \left(\int_{0}^{t}\left(B+C\|j(\tau)\|_{L^{\infty}}\right) d \tau\right) .
$$

Thanks again to Lemma 5.2 combined with (69), we deduce (55) the first part of the statement. From (67), using Lemma 5.1 and owing to inequality (55), we obtain (56), which completes the proof. 
Now, we finish with the proof of our Theorem. Assume that $\left(u_{0}, b_{0}\right) \in H^{s}\left(\mathbb{R}^{2}\right)$ with $s>2, \nabla \cdot u_{0}=0$, $\nabla \cdot b_{0}$. Thanks to Proposition 4.3, we get that there exists $T^{*}>0$ the maximal time of existence such that the MHD system of equations (3) with $\mathfrak{D}$ given by (5) has a unique local solution $(u, b)$ satisfying,

$$
(u, b) \in C\left(\left[0, T^{*}\left[; H^{s}\left(\mathbb{R}^{2}\right)\right) .\right.\right.
$$

Moreover, if $T^{*}<\infty$, then $(u, b) \notin C\left(\left[0, T^{*}\right] ; H^{s}\left(\mathbb{R}^{2}\right)\right)$. Let us assume that $T^{*}<\infty$, then thanks to Proposition 4.2, we deduce that $T^{*}$ is such that,

$$
\int_{0}^{T^{*}}\|(\omega, j)(t)\|_{L^{\infty}} d t=\infty
$$

However, using Lemmata 5.2 and 5.3, we obtain a contradiction with (70), therefore we get $T^{*}=\infty$, which concludes the proof of Theorem 1.1.

\section{Acknowledgements}

The author would like to thank the referee for his precious suggestions and corrections.

\section{References}

[1] Alfvén, H.: Existence of electromagnetic-hydrodynamic waves, Nature, 150 (1942), 3805, 405-406.

[2] Beale, J. T., Kato, T. and Majda, A.: Remarks on the Breakdown of Smooth Solutions for the 3-D Euler Equations, Comm. Math. Phys. 94 (1984), 1, 61-66.

[3] Chae, D.: Nonexistence of self-similar singularities in the viscous magnetohydrodynamics with zero resistivity, J. Funct. Anal. 254 (2008), 441-453.

[4] Chemin, J.-Y. : Perfect Incompressible Fluids, Clarendon Press, Oxford, 1998.

[5] Caflisch, R. E., Klapper, I. and Steele, G.: Remarks on singularities, dimension and energy dissipation for ideal hydrodynamics and MHD, Comm. Math. Phys. 184 (1997), 2, 443-455.

[6] Cao, C., Wu, J. and Yuan, B.: The 2D Incompressible Magnetohydrodynamics Equations with only Magnetic Diffusion, SIAM J. Math. Anal. 46 (2014), 1, 588-602.

[7] Cao, C. and Wu, J.: Global regularity for the 2D MHD equations with mixed partial dissipation and magnetic diffusion, Adv. Math. 226 (2011), 2, 1803-1822.

[8] Chen, Q., Miao, C., and Zhang, Z.: The Beale-Kato-Majda criterion for the 3D magnetohydrodynamics equations, Comm. Math. Phys. 275 (2007), 3, 861-872.

[9] Deng, J., Hou, T. Y. and Yu, X.: Geometric Properties and Non-blowup of 3-D Incompressible Euler Flow. Comm. Partial Differential Equations. 30 (2005), 1, 225-243.

[10] Fan, J., Malaikah, H., Monaquel, S., Nakamura, G. and Zhou, Y.: Global Cauchy problem of 2D generalized MHD equations, Monatsh. Math. 175 (2014), 1, 127-131.

[11] Giga, Y. and Sohr, H.: Abstract Lp estimates for the Cauchy problem with applications to the Navier-Stokes equations in exterior domains. J. Funct. Anal. 102 (1991), 1, 72-94.

[12] Jiu, Q. and Niu, D.: Mathematical results related to a two-dimensional magneto-hydrodynamic equations, Acta Math. Sci. Ser. B Engl. Ed. 26 (2006), 744-756. 
[13] Jiu, Q. and Zhao, J.: A remark on global regularity of 2D generalized magnetohydrodynamic equations, J. Math. Anal. Appl. 412 (2014), 1, 478-484.

[14] Jiu, Q. and Zhao, J.: Global regularity of 2D generalized MHD equations with magnetic diffusion, Z. Angew. Math. Phys. 66 (2015), 3, 677-687.

[15] Kato, T. and Ponce, G.: Commutator Estimates and the Euler and Navier-Stokes Equations, Comm. Pure. Applied. Math, 41 (1988), 7, 891-907.

[16] Kozono, H. and Taniuchi, Y.: Bilinear estimates and critical Sobolev inequality in BMO, with applications to the Navier-Stokes and the Euler equations, RIMS Kokyuroku, 1146 (2000), 39-52.

[17] Lei, Z. and Zhou, Y.: BKM's Criterion and Global Weak Solutions for Magnetohydrodynamics with Zero Viscosity, Discrete Contin. Dynam. Systems 25 (2009), 2, 575-583.

[18] Priest, E. and Forbes, T.: Magnetic reconnection, MHD theory and Applications. Cambridge University Press, Cambridge, (2000).

[19] Sermange, M. and Temam, R.: Some mathematical questions related to the MHD equations, Comm. Pure Appl. Math. 36 (1983), 5, 635-664.

[20] Tran, C.V., Yu, X. and Zhai, Z.: On global regularity of 2D generalized magnetodydrodynamics equations, J. Differential Equations 254 (2013), 4194-4216.

[21] Wu, J.: Generalized MHD equations, J. Differential Equations 195 (2003), 2, 284-312.

[22] Wu, J.: Regularity criteria for the generalized MHD equations, Comm. Partial Differential Equations 33 (2008), 2, 285-306.

[23] Wu, J.: Global regularity for a class of generalized magnetohydrodynamic equations, J. Math. Fluid Mech. 13 (2011), 2, 295-305.

[24] Yamazaki, K.: Remarks on the global regularity of two-dimensional magnetohydrodynamics system with zero dissipation, Nonlinear Anal., 94 (2014), 194-205.

[25] Yamazaki, K.: On the global regularity of two-dimensional generalized magnetohydrodynamics system, J. Math. Anal. Appl. 416 (2014), 1, 99-111.

[26] Ye, Z. and Xu, X.: Global regularity of the two-dimensional incompressible generalized magnetohydrodynamics system, Nonlinear Anal. 100 (2014), 86-96.

[27] Zhou, Y. and Fan, J.: A regularity criterion for the 2D MHD system with zero magnetic diffusivity, J. Math. Anal. Appl. 378 (2011), 1, 169-172. 